\title{
Non-Cavitated Approach for the Treatment of White Spot Lesions: A Case Report
}

\author{
Güvenç Başaran ${ }^{1}$, İlknur Veli ${ }^{2}$, Emine Göncü Başaran ${ }^{3}$ \\ ${ }^{1}$ Assistant Professor, Dicle University, Faculty of Dentistry, Department of Orthodontics, Diyarbakır, TURKEY \\ ${ }^{2}$ Postgraduate Student, Dicle University, Faculty of Dentistry, Department of Orthodontics, Diyarbakır, TURKEY \\ ${ }^{3}$ Research Assistant, Dicle University, Faculty of Dentistry, Department of Prosthetic Dentistry, Diyarbakır, TURKEY
}

\section{Key Words}

White spot lesion, treatment, discoloration.

\section{Correspondence: \\ Güvenç BAŞARAN \\ Dicle University, \\ Faculty of Dentistry, \\ Department of Orthodontics, \\ 21280, Diyarbakir, TURKEY. \\ e-mail: basaran@dicle.edu.tr}

\begin{abstract}
Aim: White spot lesions (WSLs) are the most frequently observed problems in patients with poor oral hygiene. If this problem is left untreated during the initial phase, it will ultimately need major treatment. One treatment alternative in the initial phase is the caries infiltration technique. The aim of this case report is to present the treatment of WSLs by using this technique.

Methodology: An 18-year-old female who had WSLs on the buccal surfaces of the lower left canine, first and second premolars and first molar after 8 months of fixed orthodontic therapy was included in this study. Icon-Infiltrant was applied for the treatment of WSLs.

Results: After Icon-Infiltrant application, the WSLs were cured. Conclusions: By using this technique, an esthetic smile can be achieved in one visit with the elimination of esthetic discoloration and the progression of the early enamel lesions can be prevented.
\end{abstract}

(Int Dent Res 2011;2:65-69)

\section{Introduction}

White spot lesions are defined as the subsurface porosity of demineralized enamel that manifests itself as milky white opacities localized on smooth surfaces $(1,2)$. The development of WSLs is a challenging problem in the course of orthodontic treatment. Especially in fixed orthodontic treatment, plaque accumulation increases around brackets and bands (3) and the pH on dental surfaces becomes acidic (4). The Streptococcus mutans (MS) and Lactobacillus (LS) populations in the plaque over the tooth surface increase and these microorganisms can cause enamel demineralization via organic acid production in the plaque $(5,6)$.

Quaard (7) observed that WSLs developed significantly more often in orthodontic patients and reported that these lesions might present severe esthetic problems in the following years. The prevalence of WSLs in patients undergoing fixed orthodontic treatment was $49.6 \%$ versus $24 \%$ in an untreated control group (8).
No gender difference was found in terms of the prevalence of WSLs (9). Gorelick et al. (8) also found no correlation between the prevalence and treatment duration. The teeth most affected by WSLs are the molars, upper laterals, lower canines, and premolars (10).

White spot lesions are the initial phase of caries lesion formation on enamel. Gorelick et al. (8) classified the lesions by into four groups: type 1 had no WSLs, type 2 had mild WSLs, type 3 had severe WSLs, and type 4 had observed cavitation. The treatment approaches for these groups change with the severity. The treatment choices for the type 2 are casein phosphopeptide-amorphous calcium phosphate (CPP-ACP)-containing mouth rinses, topical fluoride application, and fluoridated dentifrices (1). For type 3, minimally invasive approaches are the treatment of choice. The treatment options for type 4 range from the conservative treatment with Black $\mathrm{V}$ cavities to laminate veneer crowns (1).

Recently, a new technique called caries infiltration has been used for non-cavitated lesions. 
In this technique, the pore system of a noncavitated white spot or proximal lesion is filled or reinforced with a light-curable resin. "Icon", (DMG, Chemisch-Pharma. Fabrik GmbH, Hamburg) short for Infiltration Concept, is the product used with this technique for the invasive treatment of cariogenic lesions in the approximal and vestibular regions (11). Two product variants of Icon are available: Icon Caries Infiltrant-approximal was developed for incipient approximal caries and Icon Caries Infiltrant-vestibular is used for orthodontic patients after removing brackets (12).

In this case report, we present the treatment of WSLs localized on the lower left posterior region of a patient with poor oral hygiene after fixed orthodontic treatment.

\section{Case Report}

An 18-year-old female patient was referred to our clinic with esthetic and functional complaints. The patient complained of protruding upper incisors and difficulty in biting. Angle Class II dental malocclusion was observed in the intraoral examination (Fig. 1). A radiographic and periodontal examination revealed no pathological finding. A fixed orthodontic treatment with an extraoral appliance was planned. First, the upper and lower first molars were banded and Edgewise Roth brackets (Omni
Roth, GAC International, Inc, Bohemia NY, USA) with a 0.018-inch slot were bonded to them. The patient was instructed on headgear use. After an 8month active treatment period, an Angle Class I relationship was achieved on the left side. However, due to the potential for WSL occurrence and lack of oral hygiene, the patient and her parents were told about the risks and the treatment was terminated with their approval. Thus, an Angle Class II relationship remained on the right side. After the orthodontic treatment, WSLs were observed on the buccal surfaces of the lower left canine, first and second premolars, and first molar (Fig. 2a).

According to the classification of Gorelick et al. (8), the WSLs on the buccal surfaces of lower left canine, first, and second premolars were type 3, while the WSL on the buccal surface of the first molar was type 2 . After a debonding procedure with a tungsten carbide bur, the WSL on the first molar was followed only, because the lesion was type 2 and localized gingivally. The patient was informed about the treatment alternatives. She requested that the non-esthetic appearance of the canine and first and second premolars be treated as soon as possible. Considering her request, a minimally invasive, rapid treatment approach was planned with Icon. Because the WSL on the first molar was localized near the gingiva and the gingival fluid would complicate the treatment procedure, this tooth was not treated.
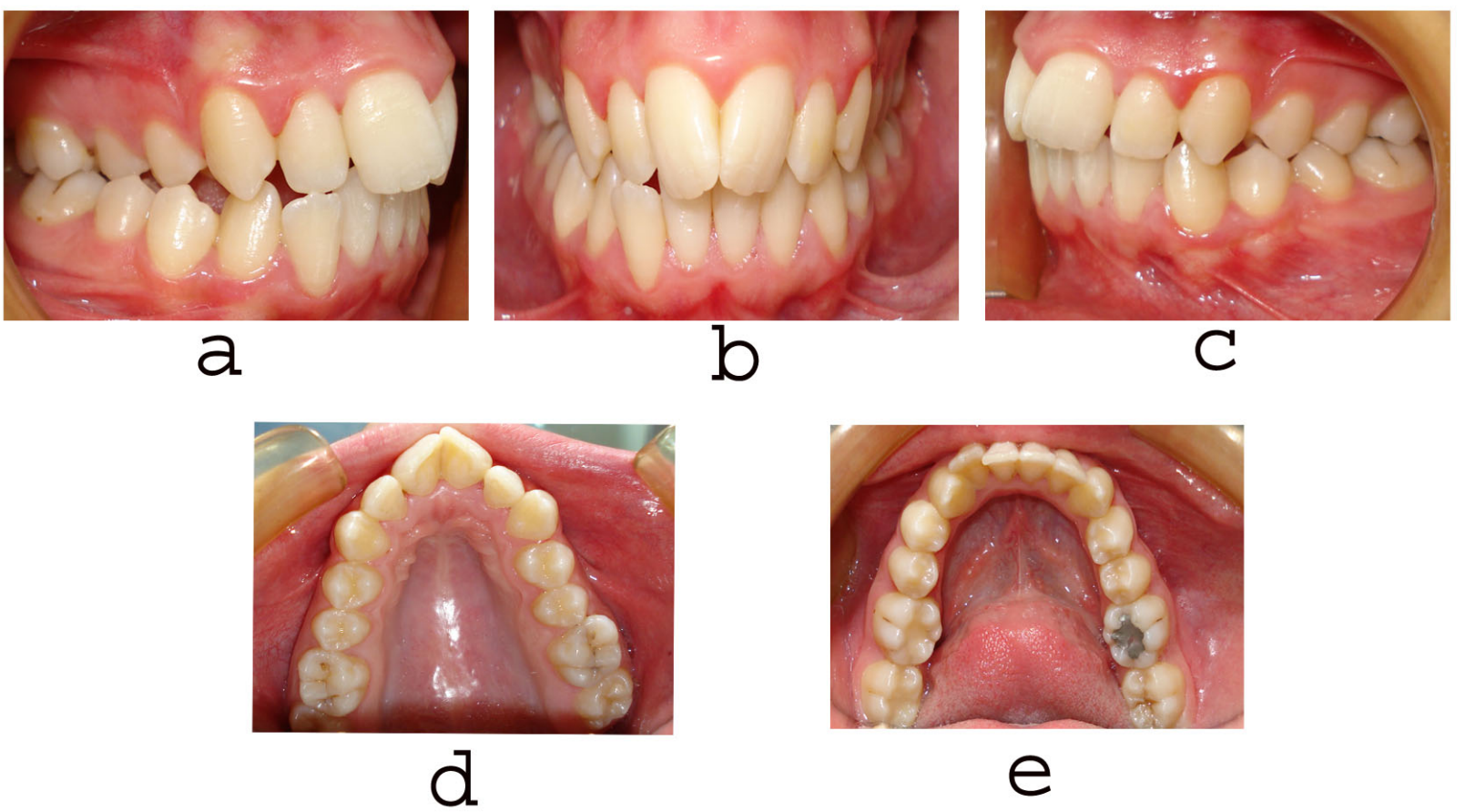

Figure 1. Pre-treatment intraoral photographs: (a) Frontal view; (b) Right view; (c) Left view; (d) Upper view; (e) Lower view. 


\section{Clinical Procedure}

After debonding, all of the teeth were polished with a non-fluoridated prophylaxis paste. A rubber dam was placed about the lower left canine and first and second premolars to provide a dry working field (Fig. 2b). The teeth surfaces were dried with oiland water-free air. A 2-mm area beyond the WSL was etched with $15 \%$ hydrochloric acid (Icon-EtchDMG,Chemisch-Pharma. Fabrik GmbH, Hamburg) for $120 \mathrm{~s}$ (Fig. 2c). Then, the $\mathrm{HCl}$ was evacuated with a surgical aspirator. The teeth surfaces were rinsed with water for $30 \mathrm{~s}$ and dried with oil- and waterfree air for $30 \mathrm{~s}$.

Next, 99\% ethanol containing Icon-Dry (DMG,Chemisch-Pharma. Fabrik GmbH, Hamburg) was applied to the dry lesion sites and allowed to set for $1 \mathrm{~min}$ (Fig. 2d). Then, the lesion sites were dried with air for $30 \mathrm{~s}$.

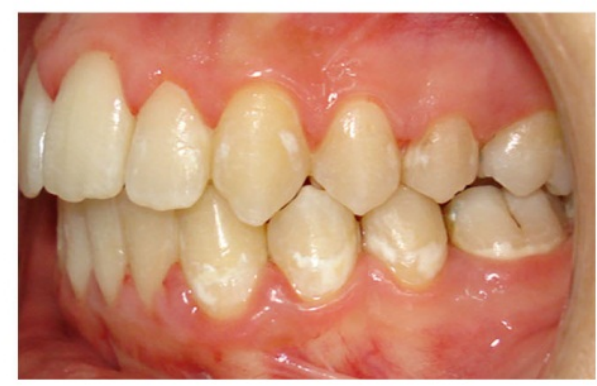

a

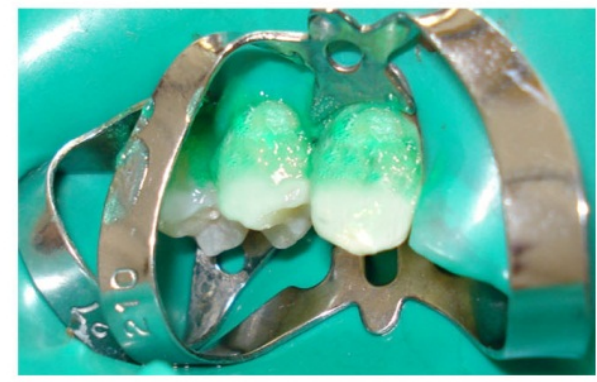

C

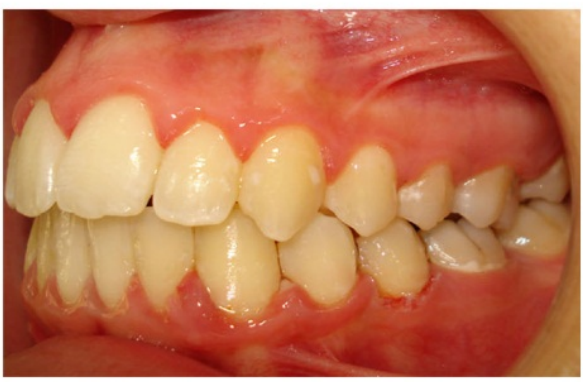

e
Icon-Infiltrant was applied on the dry, solventfree lesion sites under daylight and set for $3 \mathrm{~min}$. After $3 \mathrm{~min}$, the affected surfaces were light-cured with an Ultra-Lite 1000E LED light-curing unit (Rolence Enterprise Inc. Chungli, Taiwan) that had an output of $450 \mathrm{~nm}$ and a light intensity of 800 $\mathrm{mW} / \mathrm{cm}^{2}$ for $40 \mathrm{~s}$. The Icon-Infiltrant was applied again and allowed to set for $1 \mathrm{~min}$ and then lightcured for $40 \mathrm{~s}$. The rubber dam was removed. Irritation in the gingiva resulted from the clamp for the rubber-dam placed around the second premolar. KerrHawe finishing and polishing discs (KerrHawe $\mathrm{SA}$, Bioggio, Switzerland) were used to remove any excess Icon-Infiltrant that extended over the edges of lesion sites (Fig. 2e-f). After this treatment, the WSLs were cured and progression of the early enamel lesions was prevented. No pathological finding was observed on panoramic and cephalometric radiographs taken after Icon-Infiltrant application.

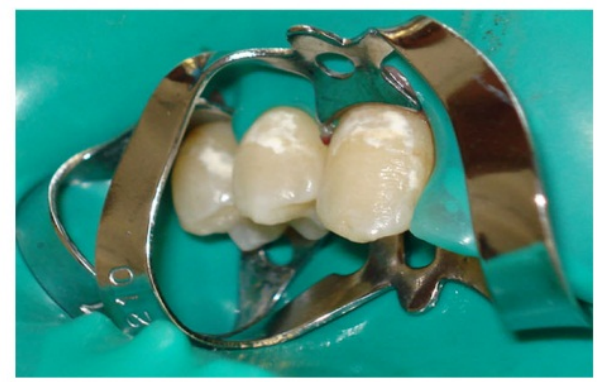

b

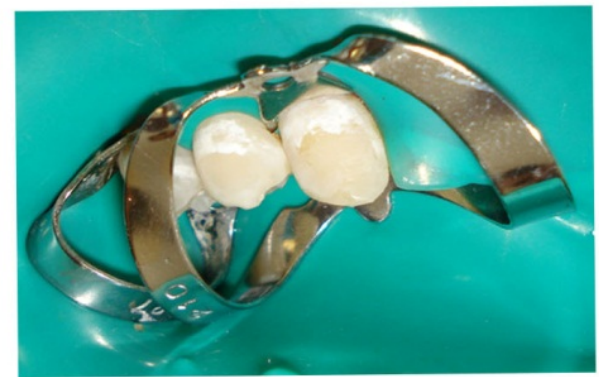

d

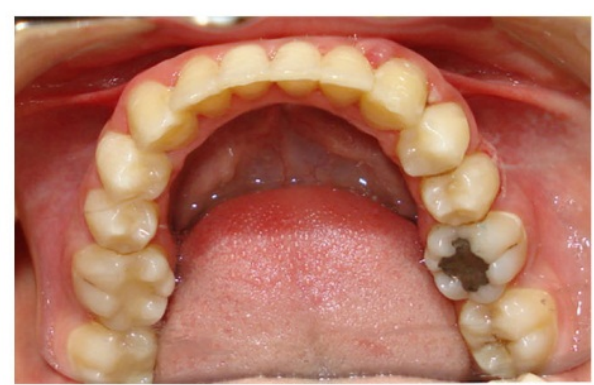

f

Figure 2. (a) Right buccal segment with WSLS after fixed orthodontic treatment; (b) Rubber-dam placement before Icon Infiltrant application; (c) The $15 \% \mathrm{HCl}$ etched teeth surfaces before Icon Infiltrant application; (d) The appearance of enamel surfaces after ethanol application; (e) The buccal appearance of teeth surfaces after Icon Infiltrant application; (f) The occlusal appearance of teeth surfaces after Icon Infiltrant application. 


\section{Discussion}

Enamel decalcification or WSLs result from the accumulation of bacterial plaque over an enamel surface. The bacterial plaque causes the surface $\mathrm{pH}$ to decrease, which disrupts the mineral balance in the enamel and decalcified areas of different shapes and sizes occur $(8,13)$. These WSLs are treated with various approaches depending on the patient and type of WSL.

Gorelick et al. (8) suggested conservative treatment approaches for types 3 and 4 WSLs. Today, WSLs are commonly prevented by good oral hygiene or fluoridation. However, in non-compliant patients, small WSLs can extend and lead to cavitation (14). Treatment with infiltration techniques that are an alternative to minimally invasive approaches and need no cavitation for noncompliant or agitated patients is preferable in terms of psychology and the rapid achievement of an esthetic outcome (15).

An innovative technique called the caries infiltration technique is used in non-cavitated approaches. Caries infiltration materials are a promising treatment for WSLs.

With the infiltration technique, a diffusion barrier is formed in the inner parts of the lesion and the affected surface is restored with resin material (14). In recent clinical research, this technique has been reported to have positive effects on $43 \%$ of proximal enamel lesion over an 18-month period (20). Other advantages include enabling the clinician to remove the excessive resin before light curing, minimal enamel loss on all surfaces, and the prevention of plaque accumulation sites by achieving smooth surfaces (14). Consequently, plaque accumulation is prevented and the periodontal structures are protected.

With this technique, $15 \% \mathrm{HCl}$ is commonly used. Sebastian et al. (14) observed that etching with phosphoric acid affected only the outermost $25 \mu \mathrm{m}$ of the surface. However, superficial phosphoric acid etching causes the caries lesion to extend into the deeper enamel layers. Consequently, cavitation may be necessary in the future.

The penetration depth of $15 \% \mathrm{HCl}$ etching is more than twice $(58 \mu \mathrm{m})$ that of phosphoric acid, enabling penetration into the deepest part of the lesion and eliminating the decalcified areas, preventing further attacks (16). A rubber dam is necessary with all applications, because $15 \% \mathrm{HCl}$ produces soft tissue ulceration within $30 \mathrm{~s}$ (17).

Several studies have reported that $120 \mathrm{~s}$ is the optimum etching time $(18,19)$. The primers and resin materials used contain solvents, such as ethanol, which increase the surface tension of enamel, enabling rapid penetration of resins into the lesions, increasing the adhesion strength $(20,21)$.

With a one-visit treatment procedure, the progression of early enamel lesions was arrested with no drilling or anesthesia. However, to evaluate the short- and long-term results, further studies should be performed with larger sample sizes.

\section{Conclusion}

This new caries infiltration technique may be an alternative for the treatment of noncompliant patients with WSLs in one visit.

\section{Acknowledgments}

The authors deny any conflicts of interest related to this study.

\section{References}

1. Bishara SE, Ostby AW. White spot lesions: Formation, Prevention and Treatment. Semin Orthod 2008;14:174-82.

2. Summit JB, Robbins JW, Schwartz RS. Fundamentals of operative dentistry: Acontemporary approach, ed 3. Hannover, Quintesentence Publishing, 2006.

3. Lovrov S, Hertrich K, Hirschfelder U. Enamel demineralization during fixed orthodontic treatment incidence and correlation to various oral hygene parameters J Orfac Orthop 2007;68: 353-63.

4. Sanpei S, Endo T, Shimooka S. Caries risk factor in children under treatment with sectional brackets. Angle Orthod 2010;8:509-14.

5. Chang HS, Walsh LJ, Frer TJ. The effect of orthodontic treatment on salivary flow, $\mathrm{pH}$, buffer capacity and levels of mutans steptococci and lactobacilli. Aust Orthod J 1999;15:229-34.

6. Rosenbloom RG, Tinanoof N. Salivary streptococus mutans levels in patients before, during and after orthodontic treatmnt. Am J Orthod Dentofacial Orthop 1991;100:35-7.

7. Qgaar B. Prevalence of white spot lesion in 19 years old: a study on unteated and orthodontically treated person 5 years after treatment. Am J Orthod Dentofacial Orthop 1989;96:423-7.

8. Gorelick L, Geiger AM, Gwinnett AJ. Incidence of white spot formation after bonding and banding Am J Orthod 1982;81:93-98. 
9. Mizrahi E. Enamel demineralization following orthodontic treatment Am J Orthod Dentofacial Orthop 1982;82:62-7.

10. Qgaar B. White spot lesions during orthodontic treatment mechanics and fluoridepreventive aspects. Semin Orthod 2008;14:183-93.

11. Glazer SH. Treating white spots: new caries infiltration technique. Dentistry today. 2009;28: 82-5.

12. www.drilling-no-thanks.com/upload/files/ download/gi_icon576_2009_09_lay.pdf. pp: 1214.

13. Sudjalim TR, Woods MG, Manton DJ. Prevention of white spot lesions in orthodontic pratice Aust Dent J 2006;51:284-9.

14. Paris S, Meyer-Luckel H, KielbassaAM. Resin Infiltration of natural caries lesion. J Dent Res 2007; $86: 662-6$.

15. Ardu S, Castioni NV, Benbachir N, Krejci I. Minimal invasive treatment of white Spot enamel lesions Quintessence Int 2007; 38:633-6.

16. Meyer-Lueckel H, Paris S. Progression of artificial enamel caries lesions after infiltration with experimental light curing resins. Caries Res 2008;42:122-8.

17. Croll TP Killian CM, Miller AS. Effect of enamel microabrasion compoud on human gingiva: report of a case. Quintessence Int 1990;21:95963.

18. Paris S,Dörfer CE, Meyer-Luckel H. Surface conditioning of natural enamel caries lesions in decidious teeth in penetration for resin infiltration. J Dent 2010;38:65-71.

19. Croll TP, Cavanaugh RR. Enamel color modification by controlled hydrocloric acid pumice abrasion. I. Technique and examples. Quintessence Int 1986;17:81-7.

20. Meyer-Lueckel $H$, Paris S, Mueller J, Cölfen $H$, Kielbassa AM. Influence of the application time on the penetration of different dental adhesives and a fissure sealent into artificial subsurface lesions in bovine enamel. Dent Mater 2006;22: 22-8.

21. Martignon S, Ekstrand KR, Ellwood R. Efficacy of sealing proximal early active lesions: an 18 month clinical study evaluated by conventional and subtraction radiography. Caries Res 2006; $40: 382-8$. 